\title{
Análise metodológica do treinamento de força como estratégia de controle da pressão arterial em idosos: uma revisão \\ (a)
}

\author{
Methodological analysis of strength training as a strategy for blood pressure \\ control in elderly: a review
}

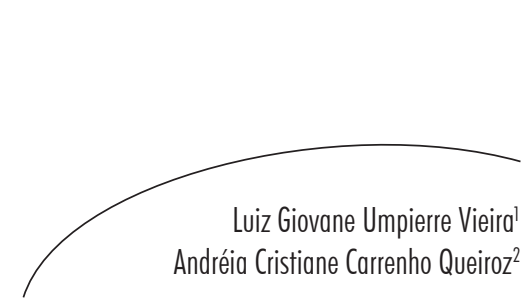

Objetivo: Realizar uma revisão sobre quais variáveis do programa de treinamento resistido estariam mais associadas com a maior redução da pressão arterial de repouso em idosos. Métodos: Foi realizado um levantamento bibliográfico nas bases de dados MEDLINE, SciELO e LILACS, compreendendo o período entre os anos 1990 e 2010, utilizando os descritores: envelhecimento, idosos, pressão arterial, exercício resistido e treinamento de força. Seguindo os procedimentos estabelecidos para o estudo, foram selecionados 16 artigos para esta revisão. Resultados: $\mathrm{O}$ treinamento resistido regular promove redução da pressão arterial de repouso em indivíduos idosos. A magnitude e a duração do efeito hipotensor variou entre os estudos, de modo que o efeito benéfico do treinamento resistido se mostrou mais evidente quando realizado com: intensidade moderada, maior número de repetições e, pelo menos, 16 semanas de duração. Conclusão: O treinamento resistido promove redução da pressão arterial, mas as características do protocolo de treinamento influenciam nesta resposta. Além disso, ainda existem controvérsias na pesquisa, e são necessárias investigações futuras.

\section{Abstract}

Aim: To assess which variables of a resistance training program could be associated with greater reduction in resting blood pressure among elderly. Methods: Literature survey was conducted in MEDLINE, SciELO a LILACS databases, covering years 1990 to 2010, using the key words: aging, elderly, blood pressure, strength exercise and resistance training. Following pre-established selection criteria, 16 articles were selected for this review. Results: Regular resistance training decreases resting blood pressure in elderly individuals. The magnitude and duration of the hypotensive effect varied among studies, so the positive effect of resistance training was more evident when training included: moderate intensity, greater number of repetitions and at least 16 weeks of duration. Conclusion: Resistance training promotes lowering blood pressure, but the characteristics of the training protocol influence this response. Moreover, there is still controversy on the subject, and further investigations are necessary.

Unidade de Campo Grande. Universidade Gama Filho. Campo Grande, MS, Brasil.

Departamento de Biodinâmica do Movimento Humano, Escola de Educação Física e Esporte. Universidade de São Paulo. São Paulo, SP, Brasil.

Correspondência / Correspondence

Luiz Giovane Umpierre Vieira

E-mail: giovane_umpierre@hotmail.com
Palavras-chave:

Envelhecimento.

Treinamento de Resistência.

Sistema Cardiovascular.

Key words: Aging. Resistance Training. Cardiovascular System. 


\section{INTRODUÇÃO}

Com o aumento da expectativa de vida, observase maior incidência e prevalência de doenças crônicas, particularmente as cardiovasculares. ${ }^{1}$ Dentre os fatores de risco cardiovasculares, a hipertensão arterial sistêmica se destaca como uma das principais doenças que afetam os idosos.

A hipertensão arterial é uma doença multifatorial. ${ }^{2}$ Seu tratamento inclui diversas intervenções, dentre elas, a prática regular de exercícios físicos, visto que a inatividade física é apontada como um colaborador para o aumento da pressão arterial (PA). ${ }^{1}$ Por outro lado, a prática de exercício físico de forma crônica promove a redução da PA. ${ }^{3}$

O treinamento resistido vem sendo recomendado como um importante componente nos programas de condicionamento para idosos, sobretudo devido às perdas musculares que ocorrem com o avanço da idade. ${ }^{4}$ Isso se deve a seus importantes benefícios, como aumento da força e potência muscular, redução da sarcopenia e melhora das capacidades funcionais no idoso.,

Além de prejuízos musculares, o envelhecimento está associado com alterações no sistema cardiovascular ${ }^{7,8}$ que podem predispor à elevação da PA de repouso, contribuindo para o aumento da prevalência de hipertensão arterial. ${ }^{8}$ Neste sentido, estudos demonstraram efeitos positivos do treinamento resistido também sobre a função cardiovascular, como redução da $\mathrm{PA}^{9-11}$ e aumento do fluxo e condutância vasculares, ${ }^{12}$ sendo este mais um motivo pelo qual esse tipo de treinamento vem sendo indicado para essa população, embora alguns resultados ainda sejam controversos. ${ }^{13}$

Segundo Polito, ${ }^{14}$ a magnitude e a duração de redução da PA após o treinamento resistido podem sofrer influência das próprias variáveis desse tipo de treinamento, como carga utilizada, número de repetições, número de séries, intervalo de repouso entre as séries, entre outras. Tais variáveis, quando manipuladas adequadamente, permitiriam atingir objetivos específicos, ${ }^{15}$ como a redução da PA de repouso.

Partindo desses pressupostos, o objetivo do estudo foi realizar uma revisão sobre quais variáveis do programa de treinamento resistido estão mais associadas com a redução da PA de repouso em idosos, entendendo os possíveis mecanismos reguladores envolvidos nessa resposta.

\section{MÉTODOS}

Para a constituição do corpus de análise desta revisão, foram realizadas buscas em bibliotecas da área de ciências biológicas, revistas eletrônicas e bases de dados virtuais, como MEDLINE (National Library of Medicine), SciELO (Scientific Electronic Library Online) e LILACS (Literatura Latino-americana e do Caribe em Ciências da Saúde). Foram utilizados os descritores em português: envelhecimento, idosos, pressão arterial, exercício resistido e treinamento de força; e seus equivalentes em inglês: aging, elderly, blood pressure, strength exercise e resistance training. Durante a busca, houve o cruzamento combinado dessas palavras. Somente artigos em inglês e português publicados entre os anos de 1990 e 2010 foram considerados para esta revisão.

Foram identificados, nas buscas, os artigos que apresentavam pelo menos duas palavraschaves inseridas em seu título e/ou resumo. Com a análise do título e do resumo, foram selecionados 45 artigos potencialmente relevantes. Estes foram lidos na integra por dois revisores de forma independente, analisando os seguintes critérios de inclusão: a) apresentar indivíduos idosos com mais de 60 anos de idade na sua amostra; b) ter investigado os efeitos crônicos do exercício resistido sobre a PA. Vinte e nove artigos não apresentaram estes critérios e, portanto, foram excluídos. Desta forma, foram selecionados 16 artigos para esta revisão. 


\section{RESULTADOS E DISCUSSÃO}

Efeito do treinamento resistido sobre a

PA de repouso em idosos

$\mathrm{Na}$ tentativa de retardar ou minimizar alguns aspectos do envelhecimento, a comunidade científica vem considerando essencial a prática do exercício físico, pois embora o envelhecimento esteja associado a diversas alterações no sistema cardiovascular, os exercícios físicos podem trazer benefícios importantes para esta população. ${ }^{10,16}$

O exercício aeróbico se destaca como importante intervenção, visto que inúmeros estudos comprovam seus benefícios agudos e crônicos sobre a estrutura e função

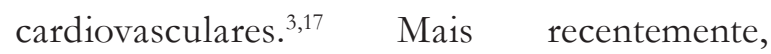
entretanto, algumas instituições de saúde, como American College of Sports Medicine ${ }^{18}$ e American Heart Association, ${ }^{19}$ começaram a recomendar que o treinamento aeróbico seja complementado pelo treinamento resistido para indivíduos com problemas cardiovasculares, sobretudo idosos, devido aos atuais indicativos dos benefícios do treinamento resistido sobre alguns fatores de risco cardiovascular e por seus inegáveis benefícios para o sistema músculoesquelético.

Com base na análise dos estudos selecionados, observaram-se diferentes respostas de PA após o treinamento resistido, possivelmente devido às diferentes características da amostra estudada e, sobretudo, os diversos protocolos de treinamentos. Segundo o quadro 1, o treinamento resistido proporcionou redução da PA de repouso, como se pode observar na maioria dos estudos (94\%). Desta forma, apenas em um estudo não houve redução na $\mathrm{PA}$ de repouso. ${ }^{12}$ Cabe ressaltar que não foi evidenciado aumento da PA de repouso em nenhum dos estudos.

Os estudos envolveram tanto idosos hipertensos quanto normotensos e, além disso, um estudo avaliou idosos diabéticos. ${ }^{20} \mathrm{O}$ total de pessoas avaliadas nos estudos selecionados foi de 565 indivíduos, com tamanhos de amostras que variaram de 12 a 70 sujeitos. Considerando os três estudos que envolveram somente indivíduos hipertensos ${ }^{21-23}$, todos observaram redução da PA de repouso. Outros cinco estudos ${ }^{9,16,20,24,25}$ apresentaram análises que incluíram sujeitos normotensos e hipertensos de forma conjunta, e todos eles observaram queda da PA de repouso. Destes, apenas o estudo de Stewart et al..$^{24}$ utilizou o treinamento aeróbico de forma concomitante ao resistido.

Vale ressaltar que a população hipertensa envolvida nas pesquisas estava sob ação medicamentosa, o que não permite avaliar o efeito do treinamento resistido isoladamente, uma vez que o uso de medicamentos pode influenciar na resposta ao exercício. Esta situação, no entanto, é a mais próxima da realidade dos indivíduos hipertensos, os quais frequentemente estão sob uso de medicação anti-hipertensiva.

E por fim, ainda em relação à característica da população estudada, dos oito estudos que envolveram apenas indivíduos normotensos, apenas o estudo de Anton et al. ${ }^{12}$ não apresentou queda da PA de repouso, enquanto nos outros sete estudos ${ }^{10,11,26-30}$ foi possível observar redução da PA, confirmando que o treinamento resistido é eficaz para reduzir a PA de indivíduos não hipertensos. ${ }^{31}$

Independentemente da população estudada, considerando os estudos que observaram redução da PA de repouso, em apenas dois o treinamento aeróbico foi realizado de forma concomitante com o resistido, ${ }^{22,24}$ fato que talvez indique que a queda da PA possa ocorrer após o treinamento resistido realizado de forma isolada em indivíduos idosos, qualquer que seja a associação desses dois tipos de treinamento. Cabe resaltar, porém, que a associação de exercícios aeróbicos e resistidos de forma combinada poderia ter um efeito hipotensor de maior magnitude. ${ }^{22}$ 
Quadro 1 - Efeito do treinamento resistido sobre a pressão arterial de idosos. 2010.

\begin{tabular}{|c|c|c|c|}
\hline Autor & Amostra & Programa de Treinamento & PAS / PAD/ PAM \\
\hline $\begin{array}{l}\text { Anton et al., } \\
2006^{12}\end{array}$ & $\begin{array}{l}26 \text { sujeitos, ambos } \\
\text { os sexos, meia } \\
\text { idade e idosos, NT }\end{array}$ & $\begin{array}{c}\text { 3x semana, } 13 \text { semanas } \\
9 \text { exercícios } \\
\text { SDS / } 12 \text { repetições } \\
75 \% \text { de } 1 \mathrm{RM}\end{array}$ & $\leftrightarrow \mathrm{PAS} / \leftrightarrow \mathrm{PAD} /$ na PAM \\
\hline $\begin{array}{l}\text { Castaneda et } \\
\text { al., } 2002^{20}\end{array}$ & $\begin{array}{c}62 \text { idosos, ambos } \\
\text { os sexos, NT, HT } \\
\text { e DM }\end{array}$ & $\begin{array}{l}\text { 3x semana, } 16 \text { semanas } \\
5 \text { exercícios } \\
3 \text { séries / } 8 \text { repetições } \\
60-80 \% \text { de } 1 \mathrm{RM}\end{array}$ & $\downarrow \mathrm{PAS} / \leftrightarrow \mathrm{PAD} /$ na PAM \\
\hline $\begin{array}{l}\text { Cononie et } \\
\text { al., } 1991^{16}\end{array}$ & $\begin{array}{l}49 \text { idosos, ambos } \\
\text { os sexos, NT e HT }\end{array}$ & $\begin{array}{c}\text { 3x semana, } 26 \text { semanas } \\
8 \text { exercícios } \\
1 \text { série / 8-12 repetições, SDI }\end{array}$ & $\begin{array}{l}\mathrm{NT}: \leftrightarrow \mathrm{PAS} / \downarrow \mathrm{PAD} / \leftrightarrow \mathrm{PAM} \\
\mathrm{HT}: \leftrightarrow \mathrm{PAS} / \leftrightarrow \mathrm{PAD} / \leftrightarrow \mathrm{PAM}\end{array}$ \\
\hline Cunha, $2010^{21}$ & $\begin{array}{l}14 \text { idosas, } \\
\text { mulheres, HT }\end{array}$ & $\begin{array}{c}\text { 3x semana, } 8 \text { semanas } \\
7 \text { exercícios } \\
\text { Prot.1: } 2 \text { séries / } 8 \text { repetições, } \\
\text { intensidade de } 8 \text { RM } \\
\text { Prot.2: } 2 \text { séries / } 16 \text { repetições, } \\
\text { intensidade de } 8 \mathrm{RM}\end{array}$ & $\begin{array}{c}\text { Prot.1: } \leftrightarrow \text { PAS } / \downarrow \text { PAD } / \downarrow \text { PAM } \\
\text { Prot.2: } \leftrightarrow \text { PAS/ } \leftrightarrow \text { PAD } / \downarrow P A M\end{array}$ \\
\hline $\begin{array}{l}\text { Delmônico et } \\
\text { al., } 2005^{9}\end{array}$ & $\begin{array}{c}70 \text { idosos } \\
\text { sedentários, ambos } \\
\text { os sexos, NT e HT }\end{array}$ & $\begin{array}{l}\text { 3x semana, } 23 \text { semanas } \\
\text { 6-8 exercícios } \\
\text { 1-2 séries / 5RM e 15RM }\end{array}$ & $\downarrow \mathrm{PAS} / \downarrow \mathrm{PAD} /$ na PAM \\
\hline $\begin{array}{l}\text { Krinski et al., } \\
2006^{22}\end{array}$ & $\begin{array}{c}53 \text { idosos } \\
\text { sedentários, ambos } \\
\text { os sexos, HT }\end{array}$ & $\begin{array}{c}\text { 3x semana, } 26 \text { semanas } \\
8 \text { exercícios } \\
3 \text { séries / } 10 \text { repetições } \\
60 \% \text { de } 1 \text { RM } \\
20 \text { min aeróbico } \\
60-70 \% \text { FCmáx }\end{array}$ & $\downarrow \mathrm{PAS} / \downarrow \mathrm{PAD} / \downarrow \mathrm{PAM}$ \\
\hline $\begin{array}{l}\text { Lovell; } \\
\text { Cuneo; } \\
\text { Gass, } 2009^{26}\end{array}$ & $\begin{array}{c}12 \text { homens idosos, } \\
\text { NT }\end{array}$ & $\begin{array}{c}\text { 3x semana, } 16 \text { semanas } \\
\text { SDE } \\
3 \text { séries / 6-10 repetições } \\
\text { 70-90\% de 1RM } \\
\end{array}$ & $\downarrow \mathrm{PAS} / \leftrightarrow \mathrm{PAD} /$ na PAM \\
\hline $\begin{array}{l}\text { Martel et al., } \\
1999^{10}\end{array}$ & $\begin{array}{l}21 \text { homens idosos, } \\
\text { sedentários, ambos } \\
\text { os sexos, NT }\end{array}$ & $\begin{array}{c}\text { 3x semana, } 24 \text { semanas } \\
7 \text { exercícios } 1-2 \text { séries / 15RM }\end{array}$ & $\downarrow \mathrm{PAS} / \downarrow \mathrm{PAD} /$ na PAM \\
\hline $\begin{array}{l}\text { Mora; Santos; } \\
\text { Saito, } 2010^{27}\end{array}$ & $\begin{array}{l}17 \text { mulheres } \\
\text { idosas, NT }\end{array}$ & $\begin{array}{l}\text { 3x semana, } 4 \text { semanas } \\
6 \text { exercícios } 2 \text { séries / } 15 \\
\text { repetições } 50 \% \text { de } 1 \mathrm{RM}\end{array}$ & $\downarrow \mathrm{PAS} / \downarrow \mathrm{PAD} /$ na PAM \\
\hline $\begin{array}{l}\text { Sallinen et al., } \\
2005^{11}\end{array}$ & $\begin{array}{l}52 \text { mulheres de } \\
\text { meia idade e } \\
\text { idosas, NT }\end{array}$ & $\begin{array}{l}\text { 2x semana, } 21 \text { semanas } \\
\text { 6-8 exercícios } 1 \text { série / 8-15 } \\
\text { repetições } 40-80 \% \text { de 1RM }\end{array}$ & $\downarrow \mathrm{PAS} / \downarrow \mathrm{PAD} /$ na PAM \\
\hline $\begin{array}{l}\text { Simons; } \\
\text { Andel, } 2006^{28}\end{array}$ & $\begin{array}{c}21 \text { idosos } \\
\text { sedentários, ambos } \\
\text { os sexos, NT }\end{array}$ & $\begin{array}{l}\text { 2x semana, } 16 \text { semanas } \\
6 \text { exercícios } 1 \text { série / } 10 \\
\text { repetições } 75 \% \text { de } 1 \mathrm{RM} \\
\end{array}$ & $\downarrow \mathrm{PAS} / \leftrightarrow \mathrm{PAD} /$ na PAM \\
\hline
\end{tabular}




\begin{tabular}{|c|c|c|c|}
\hline Autor & Amostra & Programa de Treinamento & PAS / PAD/ PAM \\
\hline $\begin{array}{l}\text { Stewart et al., } \\
2005^{24}\end{array}$ & $\begin{array}{l}53 \text { idosos, ambos } \\
\text { os sexos, NT e HT }\end{array}$ & $\begin{array}{c}\text { 3x semana, } 26 \text { semanas } \\
8 \text { exercícios } \\
2 \text { séries / 10-15 repetições, } \\
50 \% 1 \mathrm{RM} \\
\text { 45min aeróbico } \\
\text { 60-90\%FCmáx }\end{array}$ & $\leftrightarrow \mathrm{PAS} / \downarrow \mathrm{PAD} / \leftrightarrow \mathrm{PAM}$ \\
\hline $\begin{array}{l}\text { Taaffe et al., } \\
2007^{25}\end{array}$ & $\begin{array}{l}17 \text { idosos, ambos } \\
\text { os sexos, NT e HT }\end{array}$ & $\begin{array}{c}\text { 2x semana, } 20 \text { semanas } \\
7 \text { exercícios } \\
\text { Prot.1: } 1 \text { série / 8RM Prot.2: } 3 \\
\text { séries / 8RM }\end{array}$ & $\begin{array}{l}\text { Prot.1: } \leftrightarrow \text { PAS } / \downarrow \mathrm{PAD} / \leftrightarrow \mathrm{PAM} \\
\text { Prot.2: } \leftrightarrow \mathrm{PAS} / \downarrow \mathrm{PAD} / \leftrightarrow \mathrm{PAM}\end{array}$ \\
\hline $\begin{array}{l}\text { Terra et al., } \\
2008^{23}\end{array}$ & $\begin{array}{l}20 \text { mulheres } \\
\text { idosas, HT }\end{array}$ & $\begin{array}{c}\text { 3x semana, } 12 \text { semanas } \\
10 \text { exercícios } \\
3 \text { séries } / 8-12 \text { repetições } 60 \text { - } \\
80 \% \text { de } 1 \text { RM }\end{array}$ & $\downarrow \mathrm{PAS} / \leftrightarrow \mathrm{PAD} / \downarrow \mathrm{PAM}$ \\
\hline $\begin{array}{l}\text { Tsutsumi et } \\
\text { al., } 1997^{29}\end{array}$ & $\begin{array}{c}42 \text { idosos } \\
\text { sedentários, ambos } \\
\text { os sexos, NT }\end{array}$ & $\begin{array}{c}\text { 3x semana, } 12 \text { semanas } \\
12 \text { exercícios Prot.1: } 2 \text { séries / } \\
\text { 12-16 repetições } \\
\text { 55-65\% de 1RM Prot.2: } 2 \\
\text { séries / 8-12 repetições } \\
75-85 \% \text { de } 1 \mathrm{RM} \\
\end{array}$ & $\begin{array}{l}\text { Prot.1: } \downarrow \text { PAS/ } \downarrow \text { PAD/ na PAM } \\
\text { Prot.2: } \downarrow \text { PAS/↔PAD/ na PAM }\end{array}$ \\
\hline $\begin{array}{l}\text { Wood et al., } \\
2001^{30}\end{array}$ & $\begin{array}{c}36 \text { idosos, ambos } \\
\text { os sexos, NT }\end{array}$ & $\begin{array}{l}\text { 3x semana, } 12 \text { semanas } \\
8 \text { exercícios } 1-2 \text { séries / 8-15 } \\
\text { repetições } 75 \% \text { de } 5 \mathrm{RM}\end{array}$ & $\leftrightarrow \mathrm{PAS} / \downarrow \mathrm{PAD} /$ na PAM \\
\hline
\end{tabular}

PAS- pressão arterial sistólica; PAD- pressão arterial diastólica; PAM- pressão arterial média; HT- hipertensos; NT- normotensos; DM = diabéticos; na- não apresentou dados; SDS- sem dados do número de séries; SDI- sem dados sobre a intensidade do exercício; SDE- sem dados de número de exercícios; FCmáx frequência cardíaca máxima; 1RM- uma repetição máxima.

Variáveis do treinamento resistido associadas à redução da PA de repouso em idosos

Após breve análise das características dos protocolos de treinamento resistido aplicados, foi possível observar algumas evidências no que tange à redução da PA de repouso em idosos.

No que se refere à intensidade do treinamento resistido, observou-se que o treinamento realizado com alta intensidade $(70-90 \%$ de $1 \mathrm{RM}$ ), seja com normotensos ou hipertensos, proporcionou redução da PA sistólica de forma isolada. ${ }^{20,23,26,28}$ No entanto, quando o treinamento foi realizado com intensidade moderada $(50-65 \%$ de $1 \mathrm{RM})$, os estudos ${ }^{10,27,29}$ observaram redução tanto da PA sistólica como da PA diastólica. Todavia, Cunha ${ }^{21}$ demonstrou em idosos hipertensos que o treinamento realizado com maior intensidade (realizando oito repetições com carga atingida em um teste de 8RM), proporcionou redução da PA diastólica e PA média, enquanto o treinamento resistido realizado com menor intensidade (realizando 16 repetições com 50\% da carga atingida em um teste de 8RM) reduziu apenas a PA média. Tais evidências sugerem que a intensidade moderada talvez proporcione melhores resultados na redução da PA de repouso, mas este aspecto da intensidade necessita ser mais bem investigado, visto que nem todos os estudos convergem para este sentido. 
Com relação ao tempo total de treinamento, quatro estudos ${ }^{9-11,22}$ aplicaram treinamento com duração maior que 20 semanas, observandose redução tanto da PA sistólica quanto da PA diastólica. Por outro lado, o estudo que aplicou um programa de treinamento com duração total de 13 semanas $^{12}$ não mostrou redução da PA de repouso. Cabe ressaltar que a maioria dos estudos ${ }^{9-11,16,20,22,24-26,28}$ empregou períodos maiores de 16 semanas de treinamento, sugerindo que este tempo poderia ser mais eficaz para obter efeito hipotensor sobre a PA de repouso, uma vez que os indivíduos idosos necessitam de um período mais longo de treino para se adaptar aos estímulos desse treinamento. ${ }^{32}$

Quanto à frequência semanal de treinamento, três sessões por semana, realizadas de forma intercalada, foi a mais empregada nos estudos. Apenas três estudos ${ }^{11,25,28}$ utilizaram a frequência de dois dias de treinamento por semana, mas a redução da PA também foi observada. Neste sentido, independentemente da frequência semanal utilizada (duas ou três vezes por semana), o treinamento resistido promoveu hipotensão. Cabe ressaltar que não foi possível encontrar estudos que tenham realizado a comparação entre um programa de treinamento resistido de menor frequência e um programa de maior frequência semanal. Desta forma, uma frequência de duas a três sessões semanais parece suficiente para que o efeito hipotensor do treinamento resistido seja alcançado na população idosa.

Quanto ao número e ao tipo de exercício realizado, em todos os estudos selecionados foram utilizados exercícios resistidos dinâmicos (com contrações concêntricas e excêntricas), priorizando exercitar os grandes grupos musculares (dos membros superiores, inferiores e tronco), variando de cinco a 12 exercícios, com predominância de oito exercícios, por sessão de treinamento. No entanto, mesmo com esta homogeneidade do tipo de contração muscular e predominância de oito exercícios resistidos empregados por sessão, os resultados são controversos, não sendo ainda possível apontar se houve influência dessas variáveis nas respostas hipotensoras do treinamento resistido.

Quanto ao número de séries por sessão de treinamento, os estudos utilizaram de uma a três séries para cada exercício resistido. Neste sentido, com o conhecimento atual não foi possível estabelecer uma possível influência do número de séries na resposta de PA após o treinamento resistido.

O número de repetições por série, por sua vez, variou entre seis e 15, com predominância de oito a 12 repetições na maioria dos estudos. É importante ressaltar que os estudos que empregaram maior número de repetições (15) em seus programas de treinamento ${ }^{11,24,27,30}$ encontraram reduções tanto da PA sistólica quanto da diastólica. Sugeriram, desta forma, que talvez um maior volume de exercício pudesse potencializar o efeito hipotensor do treinamento resistido nos indivíduos idosos. Porém, não se pode esquecer que o volume não depende somente do número de repetições, mas também do número de exercícios e de séries por sessão de treinamento e, como mencionado, não foi possível estabelecer a relação destas duas variáveis com a maior redução da PA. Assim, mais estudos são necessários para que se possa entender o real efeito do volume de treinamento sobre a potencialização do efeito hipotensor do treinamento resistido em idoso.

Nesse contexto, observou-sequeo treinamento resistido regular proporcionou redução da $\mathrm{PA}$ de repouso em indivíduos idosos, sendo que este efeito se mostrou mais evidente com treinamento de intensidade moderada, com maior número de repetições e período de treinamento de, pelo menos, 16 semanas. Diferentes combinações das variáveis do treinamento resistido podem ser igualmente eficientes para alcançar a redução da PA de repouso em idosos, como por exemplo, o número de séries (1 a 3 séries), frequência semanal (2 a 3 vezes/semana) e número de exercícios (5 a 12 exercícios). É possível perceber, porém, que ainda existem controvérsias sobre a 
influência de algumas variáveis do treinamento resistido na redução da PA, sendo necessárias mais investigações a respeito.

\section{Prováveis mecanismos envolvidos na resposta da PA de} repouso após o treinamento resistido

Os mecanismos responsáveis pela redução da PA de repouso após o treinamento resistido em indivíduos idosos continuam sem esclarecimentos mais aprofundados, assim como em populações mais jovens. Isto se deve à carência de estudos que investiguem a questão. No entanto, existem estudos $^{11,12,16,23,25,30,33}$ que avaliaram algumas alterações ocorridas no sistema cardiovascular como resultante do efeito crônico do exercício resistido, alterações que podem estar relacionadas com a regulação da PA.

Em relação à estrutura morfológica cardíaca, após o treinamento resistido acontece uma hipertrofia fisiológica na parede do ventrículo esquerdo, provocada pelo aumento intermitente da PA que ocorre durante a realização do exercício resistido. ${ }^{34}$ Cabe ressaltar que essa sobrecarga de pressão mantém o volume da cavidade ventricular, afetando minimamente a função diastólica e sistólica, ${ }^{35}$ mas essa alteração na estrutura ventricular nem sempre tem sido observada em indivíduos idosos após o treinamento resistido. ${ }^{36}$ Deste modo, é possível que os idosos nem sempre apresentem respostas estruturais cardíacas após este tipo de treinamento de forma similar aos indivíduos mais jovens.

Terra et $\mathrm{a}^{23}$ sugerem que a queda da $\mathrm{PA}$ após o treinamento resistido seria mediada pela redução do débito cardíaco, principalmente pela diminuição do volume sistólico, que seria ocasionada pela redução do retorno venoso. No entanto, Cononie et al. ${ }^{16}$ não observaram diminuição do débito cardíaco após o treinamento resistido em idosos. Como o débito cardíaco é determinado pela frequência cardíaca e pelo volume sistólico, seria importante investigar o efeito do treinamento sobre essas variáveis em indivíduos idosos. Neste sentido, alguns estudos observaram redução do volume sistólico, ${ }^{16}$ enquanto outros observaram manutenção ${ }^{11}$, 23, 25 ou diminuição ${ }^{30}$ da frequência cardíaca após o treinamento resistido. Assim, é possível observar que ainda existem controvérsias sobre as respostas dos determinantes do débito cardíaco após o treinamento resistido em idosos e que mais investigações são necessárias para compreender este mecanismo que estaria envolvido na resposta da PA.

O treinamento resistido também pode promover efeitos na resistência vascular periférica. Neste sentido, Anton et al. ${ }^{12}$ constataram aumento do fluxo e condutância vascular na região dos membros inferiores em indivíduos de meia idade e idosos após o treinamento resistido. Em relação aos mecanismos neurais, considerando apenas os estudos com treinamento resistido que envolveram idosos, é possível observar aumento ${ }^{33}$ ou manutenção ${ }^{25}$ da atividade nervosa simpática após o treinamento. Desta forma, os mecanismos neurais após o treinamento resistido também precisam ser mais bem investigados nesta população.

Em relação à rigidez arterial, estudos realizados com adultos de diferentes faixas etárias, fisicamente treinados (treinamento resistido), quando comparados a seus pares sedentários, observaram menor complacência arterial nos indivíduos treinados..$^{37,38}$ As evidências, no entanto, sugerem que o efeito do treinamento resistido no aumento da rigidez arterial em indivíduos jovens parece ser neutralizado com a execução de exercícios aeróbicos de forma combinada. ${ }^{39} \mathrm{Não}$ se conhecem, entretanto, os mecanismos que explicam tais alterações, e a hipótese vigente parece ir na direção de possíveis alterações do conteúdo estrutural das artérias (como concentração de elastina e colágeno) após o treinamento. ${ }^{40}$ Com relação a essa resposta de rigidez arterial especificamente em indivíduos idosos, apenas o estudo de Maeda et al. ${ }^{41}$ mostrou que o treinamento resistido não promoveu modificação desta variável. Sendo assim, ainda é precoce inferir os efeitos do treinamento resistido 
sobre a rigidez arterial e se as alterações estariam relacionadas com a resposta de PA de repouso encontradas após o treinamento resistido na população idosa.

Com base nos estudos, pode-se observar que ainda é difícil explicar as respostas de PA após o treinamento resistido em indivíduos idosos, uma vez que existem poucos estudos que investigaram os possíveis mecanismos envolvidos nesta resposta. Além disso, os estudos existentes algumas vezes apresentam resultados controversos. Como limitação, é possível destacar que existem poucos estudos que investigaram os efeitos desse tipo de treinamento sobre a PA, especificamente em idosos hipertensos, problemática que é extremamente relevante.

\section{REFERÊNCIAS}

1. Miranda RD, Perrotti TC, Bellinazzi VR, Nobrega TM, Cnedoroglo MS, Toniolo Neto J. Hipertensão arterial no idoso: peculiaridades na fisiopatologia, no diagnóstico e no tratamento. Rev Bras Hipertens 2002;9(3):293-300.

2. Sociedade Brasileira de Hipertensão. VI Diretrizes Brasileiras de Hipertensão. Rev Hipertens 2010;13(1):1-68

3. Cornelissen VA, Fagard RH. Effects of endurance training on blood pressure, blood pressure-regulating mechanisms, and cardiovascular risk factors. Hypertension 2005;46(4):667-75.

4. Close JC. Prevention of falls in older people. Disabil Rehabil 2005;27(18-19):1061-71.

5. Bottaro M, Machado SN, Nogueira W, Scales R, Veloso J. Effect of high versus low-velocity resistance training on muscular fitness and functional performance in older men. Eur J Appl Physiol 2007;99(3):257-64.

6. Fiatarone MA, Marks EC, Ryan ND, Meredith CN, Lipsitz LA, Evans WJ. High-intensity strength training in nonagenarians. Effects on skeletal muscle. JAMA 1990;263(22):3029-34.

7. Lakatta EG, Levy D. Arterial and cardiac aging: major shareholders in cardiovascular disease enterprises: Part I: aging arteries: a "set up" for vascular disease. Circulation 2003;107(1):139-46.

\section{CONCLUSÃO}

De modo geral, os estudos analisados mostram que o treinamento resistido promoveu redução da pressão arterial de repouso em indivíduos idosos. Este efeito se mostrou mais evidente com treinamento resistido de intensidade moderada, com maior número de repetições e com um período de treinamento de, pelo menos, 16 semanas.

Entretanto, ainda existem controvérsias no que diz respeito a outras variáveis do treinamento, tais como número de séries, frequência semanal e número de exercícios por sessão de treinamento. Desta forma, mostra-se necessário realizar mais investigações a respeito.

8. Liao D, Arnett DK, Tyroler HA, Riley WA, Chambless LE, Szklo M, Heiss G. Arterial stiffness and the development of hypertension. The ARIC study. Hypertension 1999;34(2):201-6.

9. Delmonico MJ, Ferrell RE, Meerasahib A, Martel GF, Roth SM, Kostek MC, et al. Blood pressure response to strength training may be influenced by angiotensinogen A-20C and angiotensin II type I receptor A1166C genotypes in older men and women. J Am Geriatr Soc 2005;53(2):204-10.

10. Martel GF, Hurlbut DE, Lott ME, Lemmer JT, Ivey FM, Roth SM, et al. Strength training normalizes resting blood pressure in 65- to 73-year-old men and women with high normal blood pressure. J Am Geriatr Soc 1999;47(10):1215-21.

11. Sallinen J, Fogelholm M, Pakarinen A, Juvonen T, Volek JS, Kraemer WJ, et al. Effects of strength training and nutritional counseling on metabolic health indicators in aging women. Can J Appl Physiol 2005;30(6):690-707.

12. Anton MM, Cortez-Cooper MY, DeVan AE, Neidre DB, Cook JN, Tanaka H. Resistance training increases basal limb blood flow and vascular conductance in aging humans. J Appl Physiol (1985) 2006;101(5):1351-5.

13. Queiroz ACC, Kanegusuku H, Forjaz CLM. Efeitos do treinamento resistido sobre a pressão arterial de idosos. Arq Bras Cardiol 2010;95(1):135-40. 
14. Polito MD. Força muscular versus pressão arterial de repouso: uma revisão baseada em treinamento com pesos. Rev Bras Med Esporte 2009;15(4):299-305.

15. Simão R, Fleck SJ, Polito M, Monteiro W, Farinatti P. Effects of resistance training intensity, volume, and session format on the postexercise hypotensive response. J Strength Cond Res 2005;19(4):853-8.

16. Cononie CC, Graves JE, Pollock ML, Phillips MI, Sumners C, Hagberg JM. Effect of exercise training on blood pressure in 70- to 79-yr-old men and women. Med Sci Sports Exerc 1991;23(4):505-11.

17. Pescatello LS, Franklin BA, Fagard R, Farquhar WB, Kelley GA, Ray CA; American College of Sports Medicine. American College of Sports Medicine position stand. Exercise and hypertension. Med Sci Sports Exerc 2004;36(3):533-53.

18. American College Sports Medicine. ACSM's guidelines for exercise testing and prescription. 8th.ed. Philadelphia: Lippincott Williams \& Wilkins; 2010.

19. Williams MA, Haskell WL, Ades PA, Amsterdam EA, Bittner V, Franklin BA, et al. Resistance exercise in individuals with and without cardiovascular disease: 2007 update: a scientific statement from the American Heart Association Council on Clinical Cardiology and Council on Nutrition, Physical Activity, and Metabolism. Circulation 2007;116(5):572-84.

20. Castaneda C, Layne JE, Munoz-Orians L, Gordon PL, Walsmith J, Foldvari M, et al. A randomized controlled trial of resistance exercise training to improve glycemic control in older adults with type 2 diabetes. Diabetes Care 2002;25(12):2335-41.

21. Cunha ES. Efeito do treinamento resistido na pressão arterial e capacidade funcional de idosas hipertensas. [Dissertação de Mestrado]. Natal: Universidade Federal do Rio Grande do Norte; 2010.

22. Krinski K, Elsangedy HM, Nardo Junior N, Soares IA. Efeito do exercício aeróbio e resistido no perfil antropométrico e respostas cardiovasculares de idosos portadores de hipertensão. Acta Sci Health Sci 2006;28(1):71-6.

23. Terra DF, Mota MR, Rabelo HT, Bezerra LM, Lima RM, Ribeiro AG, et al. Reduction of arterial pressure and double product at rest after resistance exercise training in elderly hypertensive women. Arq Bras Cardiol 2008;91(5):299-305.

24. Stewart KJ, Bacher AC, Turner KL, Fleg JL, Hees PS, Shapiro EP, et al. Effect of exercise on blood pressure in older persons: a randomized controlled trial. Arch Intern Med 2005;165(7):756-62.
25. Taaffe DR, Galvão DA, Sharman JE, Coombes JS. Reduced central blood pressure in older adults following progressive resistance training. J Hum Hypertens 2007;21(1):96-8.

26. Lovell DI, Cuneo R, Gass GC. Resistance training reduces the blood pressure response of older men during submaximum aerobic exercise. Blood Press Monit 2009;14(4):137-44.

27. Mora CTR, Santos JFP, Saito VAS. Comportamento da pressão arterial e do VO2 max indireto em idosos hipertensos após exercícios resistidos. 2010, [acesso em 10 jul 2011]. Disponível em: http://de.slideshare. net/FAAblog/comportamento-da-presso-arterial-edo-vo2-max

28. Simons R, Andel R. The effects of resistance training and walking on functional fitness in advanced old age. J Aging Health 2006;18(1):91-105.

29. Tsutsumi T, Don BM, Zaichkowsky LD, Delizonna LL. Physical fitness and psychological benefits of strength training in community dwelling older adults. Appl Human Sci 1997;16(6):257-66.

30. Wood RH, Reyes R, Welsch MA, Favaloro-Sabatier J, Sabatier M, Matthew Lee C, et al. Concurrent cardiovascular and resistance training in healthy older adults. Med Sci Sports Exerc 2001;33(10):1751-8.

31. Cornelissen VA, Fagard RH, Coeckelberghs $\mathrm{E}$, Vanhees L. Impact of resistance training on blood pressure and other cardiovascular risk factors: a meta-analysis of randomized, controlled trials. Hypertension 2011;58(5):950-8.

32. Carvalho J, Soares J. Envelhecimento e força muscular - breve revisão. Rev Port Cien Desp 2004;4(3):79-93.

33. Pratley R, Nicklas B, Rubin M, Miller J, Smith A, Smith M, et al. Strength training increases resting metabolic rate and norepinephrine levels in healthy 50- to 65-yr-old men. J Appl Physiol (1985) 1994;76(1):133-7.

34. Grossman W. Cardiac hypertrophy: useful adaptation or pathologic process? American J Med 1980;69(4):576-84.

35. Colan SD. Mechanics of left ventricular systolic and diastolic function in physiologic hypertrophy of the athlete's heart. Cardiol Clin 1997;15(3):355-72.

36. Haykowsky M, Humen D, Teo K, Quinney A, Souster M, Bell G,et al. Effects of 16 weeks of resistance training on left ventricular morphology and systolic function in healthy men $>60$ years of age. Am J Cardiol 2000;85(8):1002-6. 
37. Bertovic DA, Waddell TK, Gatzka CD, Cameron JD, Dart AM, Kingwell BA. Muscular strength training is associated with low arterial compliance and high pulse pressure. Hypertension 1999;33(6):1385-91.

38. Miyachi M, Donato AJ, Yamamoto K, Takahashi K, Gates PE, Moreau KL, et al. Greater age-related reductions in central arterial compliance in resistancetrained men. Hypertension 2003;41(1):130-5.

39. Kawano H, Tanaka H, Miyachi M. Resistance training and arterial compliance: keeping the benefits while minimizing the stiffening. J Hypertens 2006;24(9):1753-9.

40. Umpierre D, Stein R. Efeitos hemodinâmicos e vasculares do treinamento resistido: implicações na doença cardiovascular. Arq Bras de Cardiol 2007;89(4):256-62.

41. Maeda S, Otsuki T, Iemitsu M, Kamioka M, Sugawara J, Kuno S, et al. Effects of leg resistance training on arterial function in older men. Br J Sports Med 2006;40(10):867-9.

Recebido: 08/3/2012

Revisado: 20/9/2012

Aprovado: 24/1/2013 